\title{
The R-Enantiomer of Ketorolac Delays Mammary Tumor Development in Mouse Mammary Tumor Virus-Polyoma Middle T Antigen (MMTV-PyMT) Mice
}

\author{
Amanda S. Peretti, ${ }^{*}$ Dayna Dominguez, ${ }^{*}$ Martha M. Grimes, ${ }^{*}$ Helen J. Hathaway, ${ }^{\dagger}$ Eric R. Prossnitz, ${ }^{\ddagger}$ Melanie R. Rivera,
} Angela Wandinger-Ness, ${ }^{\S}$ Donna F. Kusewitt, ${ }^{\S}$ and Laurie G. Hudson*

\begin{abstract}
From the Department of Pharmaceutical Sciences, ${ }^{*}$ College of Pharmacy, the Departments of Cell Biology and Physiology ${ }^{\dagger}$ and Pathology, ${ }^{\S}$ School of Medicine, and the Department of Internal Medicine, ${ }^{\ddagger}$ Division of Molecular Medicine, School of Medicine, University of New Mexico, Albuquerque, New Mexico
\end{abstract}

\author{
Accepted for publication \\ October 5, 2017. \\ Address correspondence to \\ Laurie G. Hudson, Ph.D., \\ Department of Pharmaceutical \\ Sciences, MSC09 5360, 1 \\ University of New Mexico, \\ Albuquerque, NM \\ 87131-0001. E-mail: \\ 1hudson@salud.unm.edu.
}

\begin{abstract}
Epidemiologic studies report improved breast cancer survival in women who receive ketorolac (Toradol) for postoperative pain relief compared with other analgesic agents. Ketorolac is a racemic drug. The S-enantiomer inhibits cyclooxygenases; R-ketorolac is a selective inhibitor of the small GTPases Ras-related C3 botulinum toxin substrate 1 (Rac1) and cell division control protein 42 (Cdc42), which are signaling molecules up-regulated during breast cancer progression and metastasis. The goal of this study was to determine whether R-ketorolac altered breast cancer development in the mouse mammary tumor virus-polyoma middle T-antigen model. Mice were administered ketorolac orally at $1 \mathrm{mg} / \mathrm{kg}$ twice daily to approximate the typical human dose. Mammary glands were analyzed for tumor number and immunohistochemical markers of proliferation and differentiation. R-ketorolac treatment significantly reduced mammary epithelial proliferation, based on Ki67 staining, and suppressed tumor development. Proliferative mammary epithelium from R-ketorolac-treated mice displayed greater differentiation, based on significantly higher total E-cadherin and decreased keratin 5 staining than epithelium of placebo-treated mice. No differences were detected in estrogen receptor, progesterone receptor, $\beta$-catenin, or vimentin expression between placebo and $\mathrm{R}$-ketorolac treatment groups. These findings indicate that $\mathrm{R}$-ketorolac treatment slows tumor progression in an aggressive model of breast cancer. $R$-ketorolac may thus represent a novel therapeutic approach for breast cancer prevention or treatment based on its pharmacologic activity as a Rac1 and Cdc42 inhibitor. (Am J Pathol 2018, 188: 515-524; https://doi.org/10.1016/j.ajpath.2017.10.018)
\end{abstract}

Epidemiologic evidence indicates that women who receive ketorolac (Toradol) for pain relief after breast cancer surgery have improved survival, although the basis for this effect has not been determined. ${ }^{1-5}$ Ketorolac is produced and administered as a 1:1 racemic mix of the $\mathrm{R}$ - and $\mathrm{S}$-enantiomers. The $\mathrm{S}$-form is a nonsteroidal anti-inflammatory drug that inhibits cyclooxygenase (COX) enzymes, whereas the R-form has little or no COX inhibitory activity. ${ }^{6-9}$ However, evidence is mounting that R-enantiomers of certain nonsteroidal antiinflammatory drugs are distinct chemical entities with pharmacologic activities against novel non-COX targets. ${ }^{10-13} \mathrm{We}$ previously reported that the R-enantiomer of ketorolac is an allosteric inhibitor of the Rac1 [Ras homolog (Rho)-family small GTPases Ras-related C3 botulinum toxin substrate 1] and Cdc42 (cell division control protein 42) GTPases. ${ }^{13}$ $\mathrm{R}$-ketorolac is a robust inhibitor of growth factor or serum activation of Cdc42 and Rac1, with a potency and efficacy similar to the small-molecule Cdc42 and Rac1 inhibitors, CID2950007/ML141 and NSC23766, respectively. ${ }^{13,14}$

\footnotetext{
Supported by NIH grants R21 CA170375 and 1R21CA170375-01S (L.G.H. and A.W.-N.) and Administrative Diversity Supplement to P50 GM085273 (M.R.R.). Additional support was provided by the University of New Mexico Comprehensive Cancer Center Animal Models and Human Tissue Repository Shared Resources funded by National Cancer Institute 2P30 CA118100 (principal investigator: C. Willman).

D.F.K. and L.G.H. contributed equally as senior authors.

Disclosures: A.W.-N. and L.G.H. are inventors on US patent 9,125,899 for therapeutic uses of nonsteroidal anti-inflammatory drug R-enantiomers.
} 
Furthermore, R-ketorolac, but not the S-enantiomer, inhibited Rac1- and Cdc42-dependent downstream signaling, growth factor-stimulated actin cytoskeleton rearrangements, cell adhesion, migration, and invasion in tumor cells. ${ }^{13,14}$

In many human cancers, aberrant Rho-family signaling because of changes in GTPase expression or activity is a critical contributor to tumor development and progression. ${ }^{15-22}$ Rac and Cdc42 GTPases regulate cytoskeletal dynamics important in adhesion, migration, and invasion and other cancer-relevant functions such as gene transcription, cell cycle progression, cell survival, and transformation. Several studies have demonstrated increased Rac1 and Cdc 42 activity in cancer by mechanisms that include GTPase overexpression, activating mutations, changes in expression or activity of proteins that control Rho-family GTPase activation, or changes in activity of GTPase effector molecules. ${ }^{15,23-27}$ Furthermore, Rac1 and Cdc42 knockdown or inhibition have been reported to decrease breast cancer cell growth, migration, and invasion; enhance sensitivity to ionizing radiation; and restore sensitivity to therapeutic agents such as trastuzumab and tamoxifen. ${ }^{28-36}$ These findings suggest that Rac1 and $\mathrm{Cdc} 42$ may be promising targets for clinical intervention in breast cancer. ${ }^{37}$

Given the evidence that activated Rac1 and $\mathrm{Cdc} 42$ are negative prognostic indicators in breast cancer and that ketorolac treatment may prolong survival after surgery for breast cancer, therapeutic targeting of these proteins by R-ketorolac may lead to a novel approach for breast cancer treatment. To test this hypothesis, we examined the effects of the dual Rac1 and Cdc42 inhibitor R-ketorolac on tumor progression with the use of the mouse mammary tumor virus-polyoma middle T-antigen (MMTV-PyMT) model of breast cancer. ${ }^{38}$ This genetically engineered model was selected because of its reported similarities to human disease. ${ }^{38}$ With the use of this model, we demonstrated that chronic treatment with R-ketorolac reduced proliferation of mammary epithelium, delayed mammary tumor development, and slowed mammary lesion progression.

\section{Materials and Methods}

\section{Oral Dosage Preparation}

Racemic ketorolac-tris salt was purchased from SigmaAldrich (St. Louis, MO), and individual R-enantiomer was purchased from Toronto Research Chemicals (Toronto, ON, Canada). As an alternative to gavage for oral delivery, the drug was administered in pills formed from ketorolac powder mixed into transgenic dough (BioServ, Flemington, $\mathrm{NJ}) .^{39}$ Ketorolac, as racemic compound or individual enantiomer, was stable for at least 3 months as determined by high-performance liquid chromatography.

\section{Animal Model}

FVB/N-Tg(MMTV-PyVT)634Mul/J mice, hereafter referred to as MMTV-PyMT mice, were originally obtained from
The Jackson Laboratory (Bar Harbor, ME). The colony was maintained by the University of New Mexico Comprehensive Cancer Center Small Animal Models and Imaging Shared Resource and housed at the Animal Research Facility at the University of New Mexico Health Sciences Center. Mice were maintained at a controlled temperature of $22^{\circ} \mathrm{C}$ to $23^{\circ} \mathrm{C}$, with a 12 -hour light/12-hour dark cycle. Water and standard mouse chow were available ad libitum. All procedures were approved by the University of New Mexico Institutional Animal Care and Use Committee and performed in accordance with the NIH's Guide for the Care and Use of Laboratory Animals. ${ }^{40}$

MMTV-PyMT female transgenic mice 5 to 6 weeks of age were placed into groups of two to three mice per cage and acclimated to pills by offering placebo pills twice a day for 3 days. Consumption was confirmed by direct observation. Once treatment began, mice received one pill that contained $1.0 \mathrm{mg} / \mathrm{kg}$ body weight of drug or placebo twice daily for the 3-week study or twice daily 5 days per week for the 7-week study.

\section{Tissue Analysis}

Mammary tissue whole mounts were prepared from fourth abdominal mammary glands in the 3-week study. Resected mammary glands were fixed in $4 \%$ paraformaldehyde, followed by two changes of acetone over 8 to 24 hours, then placed in water for 1 hour. After overnight staining in carmine alum $(0.2 \%$ carmine dye, $0.5 \%$ aluminum potassium sulfate), tissues were destained.

For histologic examination and immunohistochemistry of mammary tissue from the 7-week study, the fourth abdominal mammary glands were resected, fixed in neutral buffered formalin, embedded in paraffin, and sectioned longitudinally at 5 to $6 \mu \mathrm{m}$ for routine hematoxylin and eosin (H\&E) staining and immunohistochemistry. For all immunohistochemical staining, sections were rehydrated, endogenous peroxidase activity was blocked with $3 \% \mathrm{H}_{2} \mathrm{O}_{2}$ in water, and antigen retrieval was performed with $10 \mathrm{mmol} /$ L citrate buffer, $\mathrm{pH}$ 6.0, in a microwave oven. Nonspecific antibody binding was blocked with Biocare Medical Blocking Reagent (Concord, PA). Immunohistochemistry was performed with the following primary antibodies: antikeratin 5 (PRB-160P; Covance, Princeton, NJ) rabbit polyclonal diluted 1:500 for 1 hour; anti-vimentin rabbit monoclonal antibody (ab92547; Abcam, Cambridge, MA) diluted 1:500 for 1 hour; anti-E-cadherin rabbit polyclonal antibody (7870; Santa Cruz Biotechnology, Dallas, TX) diluted 1:50 for 1 hour; anti- $\beta$-catenin mouse monoclonal antibody (610153; BD Biosciences, San Jose, CA) diluted 1:500 for 30 minutes; and mouse monoclonal anti-cyclin D1 (sc-8396; Santa Cruz Biotechnology) diluted 1:500 for 2 hours. All incubations were performed at room temperature. Secondary reagents included Envision + labeled polymer, anti-rabbit-horseradish peroxidase (HRP; Dako, Santa Clara, CA) applied for 30 minutes at room temperature for 
rabbit antibodies and streptavidin-HRP (Biocare Medical) applied for 30 minutes at room temperature for mouse antibodies. Reactivity was detected using diaminobenzidine (Dako).

\section{Tumor Scoring and Morphometry}

Carmine-stained mammary gland whole mounts were imaged with MoticCam 2300 running Motic Images Plus software version 2.0 (Hong Kong, China) on an Olympus (Tokyo, Japan) SZH dissection microscope. Pixel intensity of tumor and nontumor areas was determined with ImageJ software version 1.47 (NIH, Bethesda, MD; https://imagej.nih.gov/ij). H\&E and immunohistochemistry slides were scanned by an Aperio CS2 scanner (Leica Biosystems, Buffalo Grove, IL), and morphometry was performed with the HALO image analysis platform (Indica Labs, Albuquerque, NM). Tumors were identified on H\&E-stained slides as discrete masses $>3 \mathrm{~mm}^{2}$ that had uniform structure and immunohistochemical staining characteristics and that compressed or infiltrated surrounding parenchyma. Tumor areas were determined using the manual selection tool to outline the tumors. In addition, total epithelial area in each mammary gland was determined on H\&E-stained slides by training the HALO Classifier algorithm to distinguish mammary epithelium, both hyperplastic and neoplastic, from stroma. This classifier was then applied to the entire mammary gland section. For quantification of each immunohistochemical stain, the Classifier algorithm was trained to distinguish epithelium from nonepithelial tissue; the epithelium was then analyzed with the appropriate Analysis algorithm modified as necessary for each stain.

\section{RNA Isolation and $\mathrm{qPCR}$}

Samples of tumor tissue $(30 \mathrm{mg})$ were frozen in liquid nitrogen and disrupted in buffer provided in an RNeasy Mini Kit (Qiagen, Valencia, CA), using an electric hand drill fitted with nuclease-free 1.5 -mL pestles (Kimble-Chase, Vineland, NJ). The tissue lysate was homogenized with the QIAshredder (Qiagen), and RNA was isolated with the RNeasy Mini Kit according to the manufacturer's protocols. RNA was converted into cDNA with the use of a High Capacity cDNA Reverse Transcription Kit (Applied Biosystems, Foster City, CA) and a TC-3000X Thermocycler (Techne Inc., Burlington, NJ). cDNA was generated from $1000 \mathrm{ng}$ of RNA of each sample. The resulting cDNA samples were diluted 1:3 with nuclease-free water.

Real-time quantitative PCR (qPCR) was conducted with the use of six mouse primers: Rac1, Rac1b, RhoA, Cdc42, PyMT, and $\beta-80$ actin (Qiagen; excluding PyMT; catalog numbers QT01070146, QT00127673, QT00197568, QT00091560, QT00095242, respectively). PyMT primers used were PyMT forward (5'-CGGCGGAGCGAGGAACTGAGGAGAG- $3^{\prime}$ ) and reverse (5'-TCAGAAGACTCGGCAGTCTTA- $\left.3^{\prime}\right){ }^{41}$ Fast SYBR Green Master Mix (Applied Biosystems) was used to make a 1:5 master mix for each primer. Samples were loaded in triplicate in 384-well plates with $6 \mu \mathrm{L}$ of master mix and $4 \mu \mathrm{L}$ of sample per well. A nuclease-free water sample was used as a negative control, and $\beta$-actin was included as a positive control. Genes were amplified on a 7900 HT Fast Real-Time PCR System (Applied Biosystems). Relative expression was calculated with the $\Delta \Delta$ ct method, using $\beta$-actin for normalizing and analyzing the treated samples in reference to placebo samples.

\section{Protein Isolation and Western Blot Procedures}

Proteins were extracted with frozen tumor tissues with the use of protocols modified from Zakharchenko et al. ${ }^{42}$ Briefly, 10 to $20 \mathrm{mg}$ of frozen tissue was homogenized in $250 \mu \mathrm{L}$ of RIPA buffer $(50 \mathrm{mmol} / \mathrm{L}$ Tris- $\mathrm{HCl} \mathrm{pH} 7.5,105$ $\mathrm{mmol} / \mathrm{L} \mathrm{NaCl}, 1 \% \mathrm{NP}-40,1 \%$ sodium deoxycholate, $0.1 \%$ $\mathrm{SDS}$, and $2 \mathrm{mmol} / \mathrm{L}$ EDTA) with protease inhibitors (cata$\log$ number 78439; Thermo Fisher Scientific, Waltham, MA) and phosphatase inhibitors (catalog number 78445; Thermo Fisher Scientific) with the use of an electric drill with a plastic pellet pestle (catalog number 7495150000 ; Kimble-Chase) attached. Homogenized lysates were incubated on ice for 30 minutes, mixed with a vortex mixer, and briefly sonicated. Lysates were incubated for 30 additional minutes and spun at $16,500 \times g$ for 20 minutes at $4^{\circ} \mathrm{C}$ to remove debris, and the supernatant was divided into aliquots into a new tube. Protein concentrations were measured with a Pierce BCA Protein Assay Kit (catalog number 23227; Thermo Fisher Scientific).

Proteins $(20 \mu \mathrm{g})$ were resolved by polyacrylamide gel electrophoresis and transferred to nitrocellulose membrane. Membranes were blocked in 5\% milk for 1 hour at $4{ }^{\circ} \mathrm{C}$, then blotted with the following antibodies and conditions. GTPase Western blot analyses used anti-Rac1 mouse monoclonal antibody ARC03, anti-Cdc42 mouse monoclonal antibody ACD03, anti-RhoA mouse monoclonal antibody ARH04 (Cytoskeleton, Inc., Denver, CO) at 1:500 dilution in $0.1 \%$ milk in TBST $(13 \mathrm{mmol} / \mathrm{L}$ Tris- $\mathrm{HCl}, 150$ $\mathrm{mmol} / \mathrm{L} \mathrm{NaCl}, 0.05 \%$ Tween-20 at $\mathrm{pH} 7.4$ ), RhoA antibody was diluted in TBST only. The following rabbit polyclonal antibodies from Cell Signaling (Danvers, MA) were used at 1:1000 dilution: phospho (p)-P12 (RAC1) activated kinase (PAK)1 (Ser144)/PAK2 (Ser141) antibody number 2606, PAK1 antibody number 2602, PAK1/2/3 antibody number 2604, p-p44/42 mitogen-activated protein kinase [MAPK; extracellular signal-related kinase (Erk)1/2] (Thr202/ Tyr204) antibody number 9101, p44/42 MAPK (Erk1/2) antibody number 9102, Akt antibody number 9272, and mouse monoclonal p-Akt (Ser473) (587F11) number 4051. All p-PAK, PAK, p-Erk, Erk, and Akt antibodies were diluted in 5\% bovine serum albumin in TBST, whereas pAkt was diluted in 5\% milk in TBST. Secondary antibody Anti-Rabbit IgG $(\mathrm{H}+\mathrm{L})$ HRP Conjugate W401B and AntiMouse IgG $(\mathrm{H}+\mathrm{L})$ HRP Conjugate W402B (Promega, Madison, WI) were used at 1:5000 dilution in $1 \%$ milk in TBST. Blots were stripped at $50^{\circ} \mathrm{C}$ in stripping buffer $(62.5$ 
$\mathrm{mmol} / \mathrm{L}$ Tris- $\mathrm{HCl} \mathrm{pH}$ 6.7, $100 \mathrm{mmol} / \mathrm{L} \beta$-mercaptoethanol, $2 \%$ SDS) for 30 minutes, followed by thorough rinsing with MilliQ water and three 10-minute washes in TBST. Blots were reprobed with Vinculin XP rabbit monoclonal antibody (HRP conjugate) number 18799 from Cell Signaling at a 1:1000 dilution in 5\% bovine serum albumin in TBST. Vinculin was selected because it did not change as a consequence of Rac1 overexpression in SKOV3ip cells. Western blot images were acquired on ProteinSimple FluorChem R system and analyzed with AlphaView SA software version 3.4.0.0 (ProteinSimple, San Jose, CA). Values for specific proteins were normalized to the vinculin loading control or total protein based on image analysis of Ponceau-stained membrane. ${ }^{43}$ Analysis was completed in Prism software version 6 (GraphPad Software, Inc., La Jolla, CA) with the use of unpaired $t$-tests that assumed Gaussians distributions with two-tailed $P$ values at $95 \%$ confidence intervals. To confirm no difference in variances between treatments the $\mathrm{F}$ test was used.

\section{GTPase Activity Measurement}

Rac1-GTP levels in mouse breast tumors were quantified with the use of a G-LISA kit (BK128) according to the manufacturer's instructions (Cytoskeleton, Inc.). Briefly, mouse tumors $\left(5-6 \mathrm{~mm}^{3}\right.$ in size) isolated from the left second and third mammary glands of placebo and R-ketorolac-treated mice were lysed in 700 to $900 \mu \mathrm{L}$ of the provided lysis buffer (GL36-composed of a proprietary formulation of Tris $\mathrm{pH} 7.5, \mathrm{MgCl}_{2}, \mathrm{NaCl}$, IGEPAL, and SDS), aliquots were removed for protein assay with the use of the Precision Red Advanced Protein Assay, and the remaining lysates were snap-frozen to minimize GTP hydrolysis. Lysates were thawed, and $50-\mu \mathrm{L}$ samples were immediately transferred to individual Rac1-GTP binding wells for a 30-minute incubation period at $4^{\circ} \mathrm{C}$. Wash steps were performed as described, and bound Rac1-GTP was detected with the provided anti-Rac1 primary and secondary HRP-labeled antibodies. After addition of the stop buffer, absorbances of each well were read immediately at $490 \mathrm{~nm}$ on a BioTek (Winooski, VT) Synergy Neo2 plate reader. Positive Rac1-GTP controls (100 ng constitutively active Rac1) were diluted and assayed at $3 \mathrm{ng} / \mathrm{well}$, and absorbances were used to calculate the nanogram of Rac1-GTP as a function of total milligram of protein for each sample as follows: $\left[\left(\mathrm{OD}_{\text {sample }}-\mathrm{OD}_{\text {blank }}\right) \times 3 /\left(\mathrm{OD}_{\text {Rac1-GTPstandard }}-\right.\right.$ $\left.\left.\mathrm{OD}_{\text {blank }}\right)\right] /(0.05 \mathrm{~mL} / \mathrm{mg} / \mathrm{mL}$ protein $)$. Samples for which subtraction of the blank resulted in a negative value or there was insufficient material to measure protein were not considered.

\section{Statistical Analysis}

The number of tumors per mouse, average tumor size, and total tumor area per mouse in placebo-treated versus ketorolac-treated mice were compared with the $t$-test. The number of mice with tumors in each group were compared by Fischer's exact test. HALO morphometry provided quantitative measures of total epithelial area and immunohistochemical staining for keratin 5, vimentin, E-cadherin, $\beta$-catenin, Ki-67, and cyclin D1, allowing these results to be compared with the $t$-test. All statistical analyses were performed with Prism software (GraphPad Software, Inc.).

\section{Results}

\section{R-Ketorolac Reduces Epithelial Content of Mammary Glands}

To test the impact of R-ketorolac on mammary epithelial proliferation, 5- to 6-week-old MMTV-PyMT female mice were treated with twice daily oral doses of $1.0 \mathrm{mg} / \mathrm{kg} \mathrm{R}$ ketorolac or placebo for 3 weeks. This dosing regimen achieved serum concentrations comparable with those in humans. ${ }^{8,9}$ No significant difference was found between the two treatment groups in palpable mammary nodules or body weight at sacrifice (Supplemental Figure S1). To evaluate epithelial content of the mammary glands, left and right fourth abdominal mammary glands were removed, stained with carmine alum, imaged as whole mounts, and analyzed separately. The results of the analysis were averaged for the histogram. Representative whole-mount mammary gland images for placebo versus R-ketorolac treatment groups are shown in Figure 1, A and B. Quantification of pixel intensity demonstrated a significant reduction in Carminestained epithelium in the R-ketorolac treatment group compared with the placebo group (Figure 1C). This indicated reduced epithelial volume in R-ketorolac-treated mice.

From evidence presented in Figure 1, the effects of R-ketorolac treatment were then examined on epithelial proliferation in mice with somewhat more advanced lesions. Mice received $1.0 \mathrm{mg} / \mathrm{kg}$ R-ketorolac or placebo twice daily 5 days per week for 7 weeks. No significant difference was found between placebo and R-ketorolac treatment groups in weight gain over the course of the study or in total mammary gland weight at sacrifice (Supplemental Figure S2). As determined by morphometric analysis of mammary gland sections, total epithelial area (hyperplastic and neoplastic) was significantly greater in placebo-treated mice $\left(57.96 \pm 19.13 \mathrm{~mm}^{2}\right)$ than in R-ketorolac-treated mice $\left(43.37 \pm 8.58 \mathrm{~mm}^{2}\right)$ (Figure 2, A and B). Moreover, the percentage of the mammary gland area occupied by epithelium was significantly higher in placebo-treated mice $(26.8 \% \pm 5.6 \%$ versus $21.6 \% \pm 6.5 \%)$ (Figure 2B). These findings were in agreement with the first experiment in which reduced mammary epithelium was found in mice treated with R-ketorolac for 3 weeks (Figure 1). A significant difference was found between placebo mice $(30.8 \% \pm 8.8 \%$ positive nuclei) and R-ketorolac-treated mice $(21.9 \% \pm 11.1 \%$ positive nuclei) in Ki67 staining 

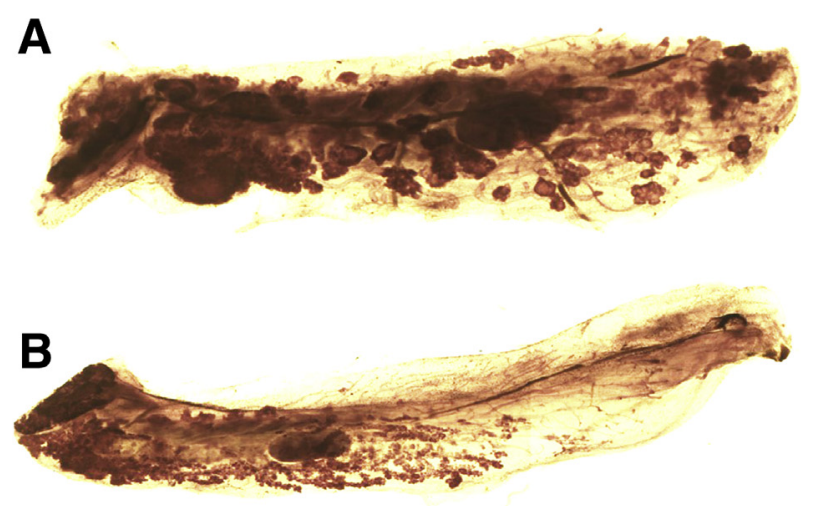

C

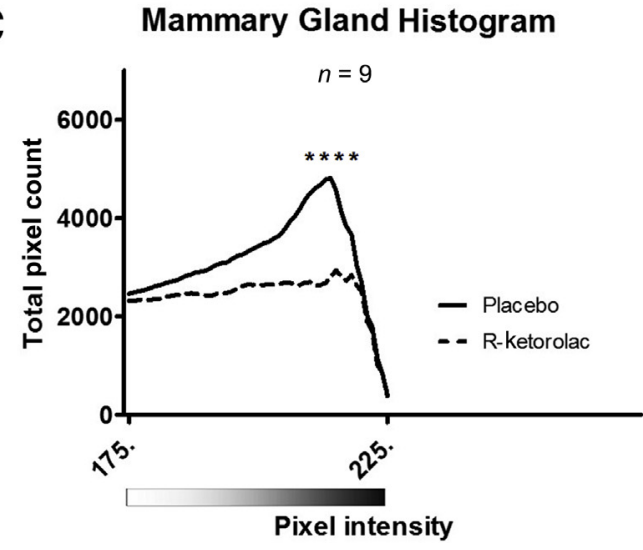

Figure 1 Three-week treatment with R-ketorolac reduces epithelial area. $\mathbf{A}$ and $\mathbf{B}$ : Carmine staining of mammary gland whole mounts obtained from mice after 3-week dosing with placebo (A) or R-ketorolac (B). Image analysis was performed on left and right fourth mammary glands; the values were averaged for each mouse with nine mice per treatment group. Darker pixels represent epithelial tissue, whereas lighter pixels represent more fatty tissue. C: Pixel intensity throughout the glands was measured with ImageJ version 1.47 software (NIH, Bethesda, MD; https://imagej.nih.gov/ ij). Placebo-treated mice had significantly greater density of staining in the mammary glands than mice treated with R-ketorolac. ${ }^{* * * *} P<0.0001$. Significance was determined with the $t$-test.

(Figure 2, C and D), but no significant change in nuclear cyclin D1 staining between the groups (Supplemental Figure S3). These findings indicated that R-ketorolac treatment reduced epithelial proliferation.

\section{R-Ketorolac Slows Tumor Development}

The mammary glands of mice in the 7-week study contained a complex admixture of epithelial lesions, including hyperplasia, adenomas, and carcinomas, as well as intermediate lesions; there were few advanced carcinomas. This made identification and classification of individual tumors difficult. Thus, stringent criteria were applied to reproducibly distinguish tumors from surrounding lesions (see Materials and Methods). Results showed that significantly fewer R-ketorolac-treated mice had tumors than placebotreated mice (Figure 3A). Although 100\% of placebotreated mice had one or more discrete mammary tumors, only $50 \%$ of R-ketorolac-treated mice had similar tumors. The mean number of tumors per mouse, when all mice were considered, was significantly lower in R-ketorolac-treated mice $(0.90 \pm 0.99$ tumors per mouse) than in placebotreated control mice $(1.73 \pm 0.79$ tumors per mouse) (Figure 3B). However, when only mice with tumors were considered, no significant difference was found between Rketorolac-treated and placebo-treated mice in number of tumors per mouse, average tumor size, or total tumor area per mouse (Supplemental Figure S4). These findings suggested that R-ketorolac decreased mammary tumor development in MMTV-PyMT mice based on number of mice developing tumors but did not reduce tumor size or area once tumors were present.

\section{R-Ketorolac Does Not Alter GTPase or PyMT Transcript Expression but Decreases GTPase Protein Levels}

Expression of the PyMT transcript, as measured by qPCR, was not altered in the mammary glands of R-ketorolac-treated mice (Supplemental Figure S5). This indicated that reduced expression of PyMT in the mammary glands of R-ketorolactreated mice was not responsible for decreased tumor development. Similarly, R-ketorolac did not alter transcripts of the Rho-family GTPases, Rac1, a constitutively active variant Rac1b, Cdc42, or RhoA in the mammary glands of ketorolactreated mice (Supplemental Figure S5). In contrast to the mRNA findings, a significant decrease in Rac1 protein levels and trend toward decrease in $\mathrm{Cdc} 42$ protein levels in tumors from R-ketorolac-treated mice compared with placebo control mice was detected (Supplemental Figure S6A). A trend was found toward a corresponding decrease in Rac1 activity (Supplemental Figure S6B). The decrease in the R-ketorolac target GTPases Rac1 and Cdc42 was selective with neither a decrease in the related family member RhoA (Supplemental Figure S6A) nor in total ERK or AKT proteins (Supplemental Figure S7). R-ketorolac decreased AKT signaling as detected by decreased phosphorylated AKT that may account, in part, for the observed reduction in epithelial proliferation in R-ketorolac-treated mice. Rac1 degradation has been reported ${ }^{43-45}$ and highlighted the complexity of mechanisms that regulate Rac1.

\section{R-Ketorolac Treatment Delays Lesion Progression}

The MMTV-PyMT mouse model of breast cancer displays many hallmarks of human breast cancer development and progression, ${ }^{38}$ including that progression associated with epithelial-to-mesenchymal transition (EMT). To determine the effect of R-ketorolac on lesion progression, immunohistochemistry and morphometry were used to measure expression of select EMT markers in proliferative mammary epithelium from MMTV-PyMT mice on the 7-week study. Staining for the epithelial marker E-cadherin was almost entirely restricted to cell margins. This staining was quantified as the number of positive pixels per squared millimeter 

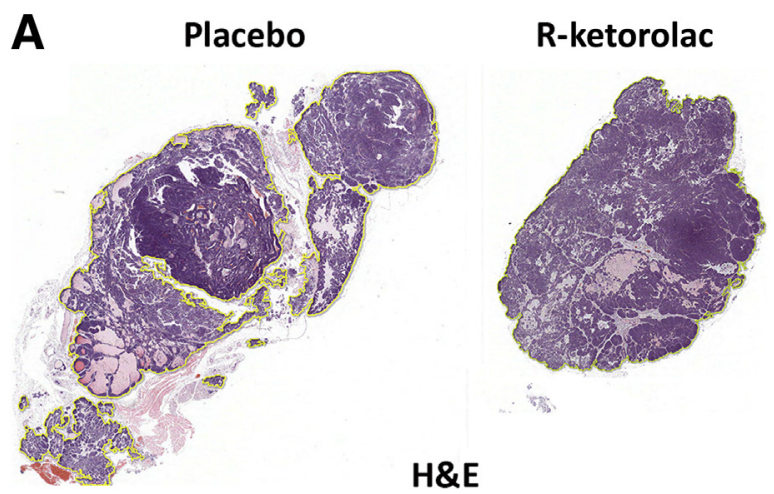

B
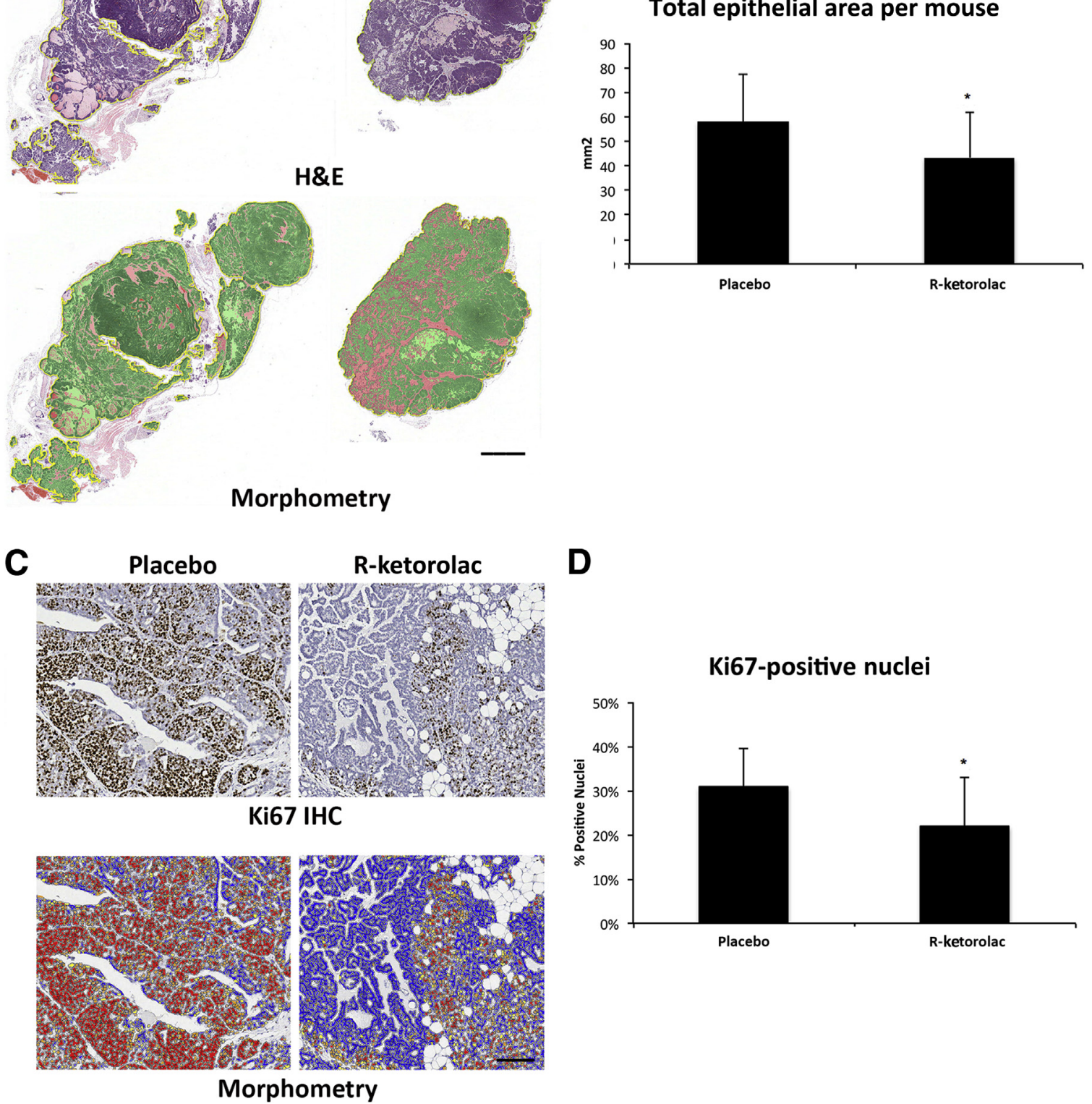

D

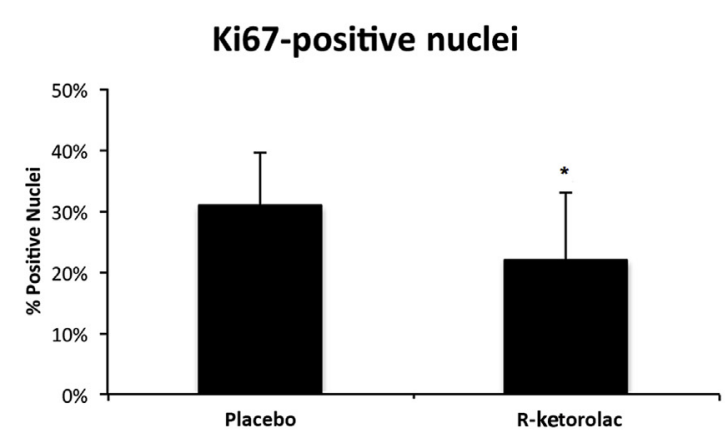

Figure 2 Seven-week treatment with R-ketorolac reduces epithelial proliferation. A: Comparison of mammary glands in mice receiving placebo or R-ketorolac for 7 weeks. Upper panels show examples of mammary glands stained with hematoxylin and eosin (H\&E), and lower panels show morphometric analyses of the same images performed to quantify total epithelial area. B: Quantification of epithelial area of the fourth mammary gland from each mouse treated with placebo or R-ketorolac shown as total epithelial area. Ketorolac-treated mice had smaller amounts of mammary epithelial tissue than placebotreated mice. C: Representative images of Ki67 immunostaining (upper panels) and corresponding morphometric analyses (lower panels) used to quantify Ki67-positive nuclei. D: Quantification of Ki67-positive nuclei from the fourth mammary gland from each mouse treated with placebo or R-ketorolac. Only the nuclei with the strongest staining (shown in red) were included. Fewer Ki67-positive nuclei in ketorolac-treated versus placebo-treated mice indicate reduced epithelial proliferation in ketorolac-treated mice. ${ }^{*} P<0.05$. Significance was determined with the one-tailed $t$-test. Scale bars: $2 \mathrm{~mm}(\mathbf{A}) ; 100 \mu \mathrm{m}(\mathbf{C})$. IHC, immunohistochemistry.

of mammary epithelium (Figure 4, A and B). Significant maintenance of staining for E-cadherin was found in the mammary glands of mice treated with R-ketorolac $\left(2.83 \pm 0.67 \times 10^{5}\right.$ positive pixels $\left./ \mathrm{mm}^{2}\right)$ compared with placebo $\left(2.33 \pm 0.35 \times 10^{5}\right.$ positive pixels $\left./ \mathrm{mm}^{2}\right)$. However, no difference was found between treatment groups in staining for the progression markers $\beta$-catenin and cytoplasmic vimentin (Supplemental Figure S8). 


\section{A Mice with tumors}

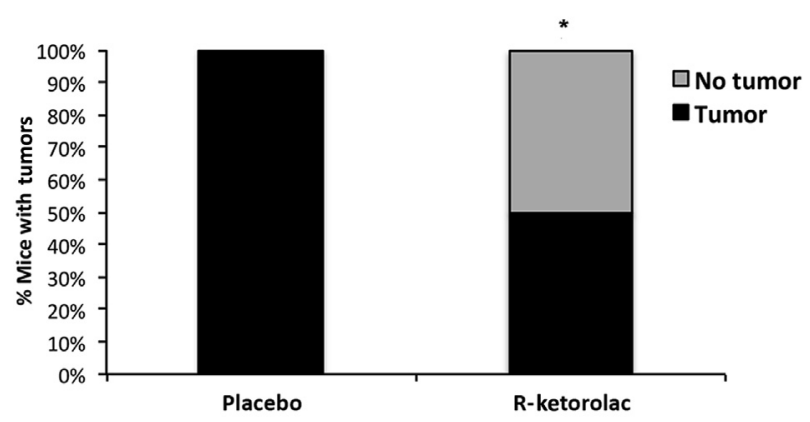

B Tumors per mouse with all mice considered

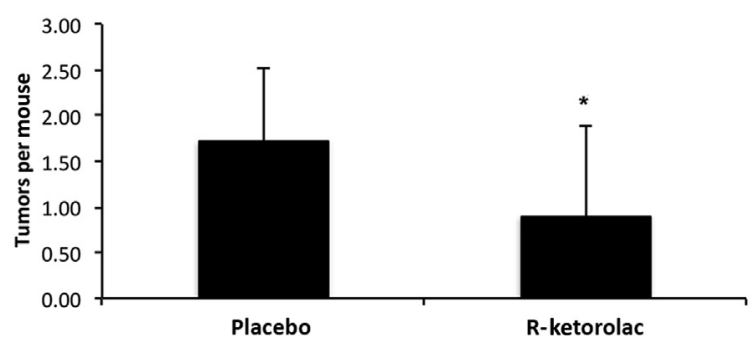

Figure 3 R-ketorolac slows mammary tumor development. A: Fourth mammary glands from mice treated with placebo or R-ketorolac were analyzed to distinguish tumors from other epithelial lesions as described in Materials and Methods. Fewer mice receiving R-ketorolac for 7 weeks develop tumors compared with placebo-treated mice. B: The number of tumors per mouse was determined as in A. All mice, including those with and without tumors, were represented in this analysis. Treatment with Rketorolac reduces the number of tumors per mouse. ${ }^{*} P<0.05$. Significance was determined by Fischer's exact test.

The MMTV-PyMT model is generally considered to represent a luminal type of mammary cancer, and mammary epithelial cells in this model predominantly express keratin 8 . However, when polyoma middle $\mathrm{T}$ antigen or ErbB2 signaling is activated in these cells, more keratin 5-positive cells appear, indicating a more basal phenotype. ${ }^{46}$ Increased expression of keratin 5 in human breast tumors is associated with poorer prognosis and enhanced resistance to therapy. ${ }^{47,48}$ Because of the large size and irregular contour of keratin 5-positive cells in MMTV-PyMT mice, the number of keratin 5-positive cells was estimated by measuring keratin 5 staining per squared millimeter of mammary epithelium. In R-ketorolac-treated mice significantly less keratin 5 staining was found than in placebo-treated mice $\left(3.13 \pm 029 \times 10^{4}\right.$ versus $3.60 \pm 0.42 \times 10^{4}$ positive pixels $/ \mathrm{mm}^{2}$ epithelium) (Figure 4, C and D), indicative of a less basal phenotype.

Advanced tumors in MMTV-PyMT mice contain fewer estrogen receptor- $\alpha$-positive $\left(\mathrm{ER}^{+}\right)$and progesterone receptor positive $\left(\mathrm{PR}^{+}\right)$cells than less advanced lesions. ${ }^{38}$ Because of the relatively early stage of the lesions in our study, the number of epithelial cells with $\mathrm{ER}^{+}$and $\mathrm{PR}^{+}$nuclei was determined in the entire mammary gland, rather than in individual tumors. $\mathrm{R}$-ketorolac treatment did not alter the total number of $\mathrm{ER}^{+}$or
$\mathrm{PR}^{+}$cells in the mammary epithelium compared with placebo treatment (Supplemental Figure S9). Although there were more tumors in placebo-treated mice than in R-ketorolactreated mice, this difference was not reflected in an overall loss of ER or PR expression. This was consistent with the observation that few advanced carcinomas were present either in placebo-treated or R-ketorolac-treated mice.

Taken together, these findings indicated that R-ketorolac decreased selected aspects of lesion progression in the MMTV-PyMT model of human breast cancer. Although Rketorolac treatment resulted in E-cadherin retention, presumably representing decreased EMT, it did not alter expression of the other EMT markers $\beta$-catenin and vimentin. Furthermore, reduced numbers of keratin 5-positive cells in the mammary glands of R-ketorolac-treated mice suggested reduced metastatic potential and decreased likelihood of treatment resistance.

\section{Discussion}

The Rho-family GTPases Rac1 and Cdc42 are key regulators of the actin cytoskeleton reorganization necessary for normal cell adhesion and migration, and are signaling molecules that modulate broad aspects of cancer cell function. ${ }^{15-22}$ Our previous studies identified R-ketorolac as a dual Rac1 and Cdc42 inhibitor with the use of a combination of high-throughput screening and computational simulation. ${ }^{13}$ R-ketorolac is one enantiomer of a racemic drug (Toradol) approved by the Food and Drug Administration for pain relief. Because epidemiologic studies indicate survival advantage for breast cancer patients who received racemic ketorolac postoperatively, ${ }^{1-5}$ the potential benefits of the R-enantiomer alone were assessed on the development and progression of early mammary gland lesions in the MMTV-PyMT mouse model of breast cancer that recapitulates many key features of human disease. ${ }^{38}$

The impact of Rac1 and Cdc42 activity on breast cancer development has not been previously studied in the MMTVPyMT model, but there are similarities between our findings and results reported in other in vivo studies that used genetic or xenograft models. Inhibition of Rac1 by the inhibitor EHop-016 in a xenograft model that used MDA-MB-435 mammary tumor cells decreased tumor growth and angiogenesis. ${ }^{49}$ Knockdown of $\mathrm{Cdc} 42$ modestly decreased growth of MDA-MB-231 cells in vivo, and pretreatment of tumor cells with the Cdc42 selective inhibitor ML141 decreased the number of mice that developed tumors. ${ }^{35}$ Genetic ablation of T-cell lymphoma invasion and metastasis 1, a Rac1-activating guanine nucleotide exchange factor, delayed tumor development, decreased the number of tumors per mouse, and led to an increase in tumor-free mice in an MMTV-c-neu breast cancer model. ${ }^{50}$ Similarly, knockout of dedicator of cytokinesis 1, another Rac1 guanine nucleotide exchange factor, in a breast cancer model that depended on human epidermal growth factor receptor 2 

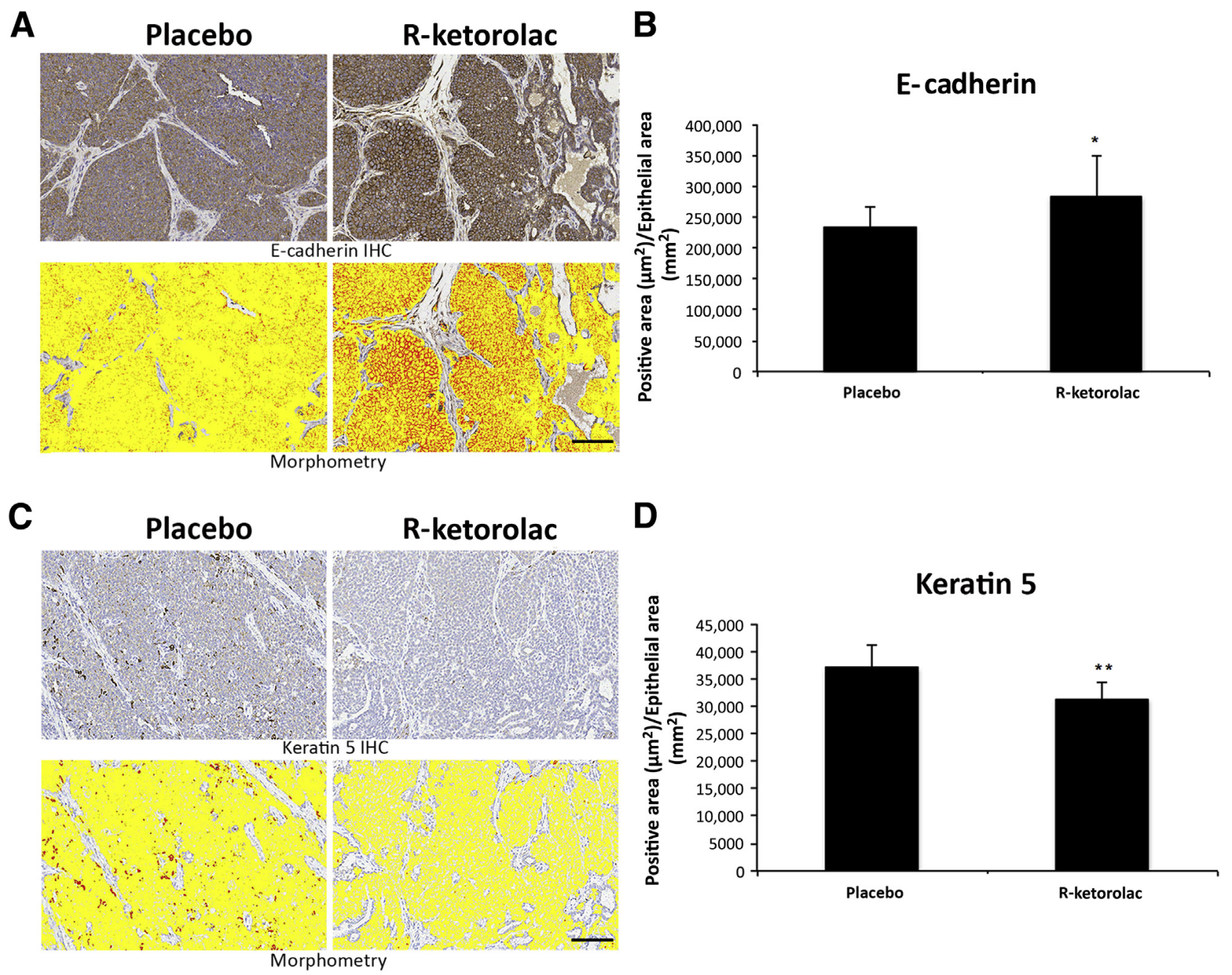

Figure 4 R-ketorolac delays mammary lesion progression. A: Representative images for E-cadherin immunostaining (upper panels) and corresponding morphometric analysis (lower panels) used to quantify E-cadherin staining. B: Quantification of E-cadherin staining from the fourth mammary gland from each mouse treated with placebo or R-ketorolac. Significantly higher E-cadherin staining, indicating reduced epithelial-to-mesenchymal transition, was found in R-ketorolac-treated mice. C: Representative images for keratin 5 immunostaining (upper panels) and corresponding morphometric analysis (lower panels) used to quantify cytokeratin 5. D: Quantification of cytokeratin 5 staining from the fourth mammary gland from each mouse treated with placebo or R-ketorolac. Significantly less keratin 5 staining, suggesting slowed lesion progression, was found in ketorolac-treated mice. Results are shown as the total positive pixel area $\left(\mu \mathrm{m}^{2}\right)$ per total epithelial area $\left(\mathrm{mm}^{2}\right)$. ${ }^{\star} P<0.05,{ }^{*} P<0.01$. Significance was determined with the one-tailed $t$-test. Scale bars $=100 \mu \mathrm{m}$. IHC, immunohistochemistry.

decreased mammary intraepithelial lesions and decreased Ki67 $7^{+}$tumor cells by approximately $30 \% .^{51}$ These two studies of genetic disruption of the Rac1 signaling pathway in breast cancer models driven by human epidermal growth factor receptor 2 are consistent with the outcomes of pharmacologic inhibition by R-ketorolac that were observed in MMTV-PYMT mice shown here.

The present study provides additional insights into the potential value of Rac1 and Cdc42 inhibition by R-ketorolac in breast cancer prevention or treatment. Decreased tumor development in R-ketorolac-treated mice was accompanied by retention of epithelial characteristics as determined by the persistence of E-cadherin expression, and reduced basal cell characteristics, as indicated by decreased keratin 5-expressing cells. In studies of MMTV-PyMT tumor organoids, cells with a basal phenotype were shown to be invasive. ${ }^{52}$ In the present study, R-ketorolac treatment is likely to contribute to reduced invasiveness by reducing the number of keratin 5-positive cells with a basal phenotype. In the MMTV-PyMT model, reduced E-cadherin and $\beta$-catenin expression and de novo expression of vimentin in mammary tumor cells are associated with EMT and increased tumor invasiveness and metastasis. ${ }^{53-56}$ Of interest, loss of Ecadherin in MMTV-PyMT mammary epithelium during mammary carcinogenesis appears to be Rac 1 dependent. ${ }^{56}$ Loss of E-cadherin is generally the earliest sign of EMT $^{57}$; thus, it is possible that similar staining for $\beta$-catenin and vimentin in R-ketorolac- and placebo-treated mice reflected the relatively early stage of mammary lesion development in these samples. Taken together, our observations indicate that ketorolac treatment reduced the progression and invasive potential of mammary gland lesions 
and motivates future studies in other models to further delineate the effects and benefits of Rac1 and Cdc42 inhibition on breast cancer development and progression.

The present and earlier studies on the antitumor effects of Rac1 and/or Cdc42 inhibition support further investigations of $\mathrm{R}$-ketorolac as a novel therapeutic approach in breast cancer. Indeed, there are active efforts to identify Rac1 and Cdc42 inhibitors, but the agents identified thus far have not been translated to human use. ${ }^{18-20,23,32,36,37,47,58}$ Retrospective studies demonstrated breast and ovarian cancer survival benefit in patients who received racemic ketorolac for indication of pain management as approved by the Food and Drug Administration, ${ }^{1-5,59}$ but toxicity risks associated with COX inhibition by the S-enantiomer limit racemic ketorolac use to 5 days. The limits are due to the gastrointestinal and cardiovascular side effects and to renal toxicity that are generally attributed to the potent COX inhibitory activities of S-ketorolac (Toradol Oral ketorolac tromethamine tablets; F. HoffmannLaRoche, Basel, Switzerland ${ }^{60}$ ). The lack of COX inhibition by R-ketorolac ${ }^{9,10,15}$ and the demonstrated inhibition of Rac1 and $\mathrm{Cdc} 42$ by racemic ketorolac in humans ${ }^{59}$ suggest that R-ketorolac may offer a tractable option for targeting Rac1 and $\mathrm{Cdc} 42$ in human disease through rapid repurposing and testing in clinical prevention or treatment trials.

\section{Acknowledgments}

Frederick Schultz (Human Tissue Repository and Tissue Analysis Shared Resource, Department of Pathology and Comprehensive Cancer Center, University of New Mexico) and Nancy Otto (Histology \& Tissue Processing Facility Core, The Virginia Harris Cockrell Cancer Research Center, MD Anderson Cancer Center, University of Texas) made important contributions to this study.

\section{Supplemental Data}

Supplemental material for this article can be found at https://doi.org/10.1016/j.ajpath.2017.10.018.

\section{References}

1. Forget P, Vandenhende J, Berliere M, Machiels JP, Nussbaum B, Legrand C, De Kock M: Do intraoperative analgesics influence breast cancer recurrence after mastectomy? A retrospective analysis. Anesth Analg 2010, 110:1630-1635

2. Retsky M, Rogers R, Demicheli R, Hrushesky WJ, Gukas I, Vaidya JS, Baum M, Forget P, Dekock M, Pachmann K: NSAID analgesic ketorolac used perioperatively may suppress early breast cancer relapse: particular relevance to triple negative subgroup. Breast Cancer Res Treat 2012, 134:881-888

3. Retsky M, Demicheli R, Hrushesky WJ, Forget P, De Kock M, Gukas I, Rogers RA, Baum M, Sukhatme V, Vaidya JS: Reduction of breast cancer relapses with perioperative non-steroidal anti-inflammatory drugs: new findings and a review. Curr Med Chem 2013, 20:4163-4176

4. Forget P, De Kock M: Perspectives in anaesthesia for cancer surgery. J Cancer Res Clin Oncol 2014, 140:353-359
5. Forget P, Bentin C, Machiels JP, Berliere M, Coulie PG, De Kock M: Intraoperative use of ketorolac or diclofenac is associated with improved disease-free survival and overall survival in conservative breast cancer surgery. Br J Anaesth 2014, 113:i82-i87

6. Kean WF, Lock CJ, Howard-Lock HE: Chirality in antirheumatic drugs. Lancet 1991, 338:1565-1568

7. Carabaza A, Cabré F, Rotllan E, Gómez M, Gutiérrez M, García ML, Mauleón D: Stereoselective inhibition of inducible cyclooxygenase by chiral nonsteroidal antiinflammatory drugs. J Clin Pharmacol 1996, 36:505-512

8. Mroszczak E, Combs D, Chaplin M, Tsina I, Tarnowski T, Rocha C, Tam Y, Boyd A, Young J, Depass L: Chiral kinetics and dynamics of ketorolac. J Clin Pharmacol 1996, 36:521-539

9. Jett MF, Ramesha CS, Brown CD, Chiu S, Emmett C, Voronin T, Sun T, O'Yang C, Hunter JC, Eglen RM, Johnson RM: Characterization of the analgesic and anti-inflammatory activities of ketorolac and its enantiomers in the rat. J Pharmacol Exp Ther 1999, 288:1288-1297

10. Yasui H, Hideshima T, Hamasaki M, Roccaro AM, Shiraishi N, Kumar S, Tassone P, Ishitsuka K, Raje N, Tai YT, Podar K, Chauhan D, Leoni LM, Kanekal S, Elliott G, Munshi NC, Anderson KC: SDX-101, the R-enantiomer of etodolac, induces cytotoxicity, overcomes drug resistance, and enhances the activity of dexamethasone in multiple myeloma. Blood 2005, 106:706-712

11. Kolluri SK, Corr M, James SY, Bernasconi M, Lu D, Liu W, Cottam HB, Leoni LM, Carson DA, Zhang X: The R-enantiomer of the nonsteroidal antiinflammatory drug etodolac binds retinoid $\mathrm{X}$ receptor and induces tumor-selective apoptosis. Proc Natl Acad Sci U S A 2005, 102:2525-2530

12. Inoue $\mathrm{T}$, Murano M, Yoda $\mathrm{Y}$, Kuramoto T, Kakimoto K, Ishida K, Kawakami K, Abe Y, Morita E, Murano N, Tokioka S: R-etodolac induces E-cadherin and suppresses colitis-related mouse colon tumorigenesis. Oncol Rep 2010, 24:1487-1492

13. Oprea TI, Sklar LA, Agola JO, Guo Y, Silberberg M, Roxby J, Vestling A, Romero E, Surviladze Z, Murray-Krezan C, Waller A, Ursu O, Hudson LG, Wandinger-Ness A: Novel activities of select NSAID R-enantiomers against Rac1 and Cdc42 GTPases. PLoS One 2015, 10:e142182

14. Guo Y, Kenney SR, Muller CY, Adams S, Rutledge T, Romero E, Murray-Krezan C, Prekeris R, Sklar LA, Hudson LG, WandingerNess A: R-ketorolac targets Cdc42 and Rac1 and alters ovarian cancer cell behaviors critical for invasion and metastasis. Mol Cancer Ther 2015, 14:2215-2227

15. Alan JK, Lundquist EA: Mutationally activated Rho GTPases in cancer. Small GTPases 2013, 4:159-163

16. Orgaz JL, Herraiz C, Sanz-Moreno V: Rho GTPases modulate malignant transformation of tumor cells. Small GTPases 2014, 5:e29019

17. Ridley AJ: Rho GTPase signalling in cell migration. Curr Opin Cell Biol 2015, 36:103-112

18. Pajic M, Herrmann D, Vennin C, Conway JR, Chin VT, Johnsson AK, Welch HC, Timpson P: The dynamics of Rho GTPase signaling and implications for targeting cancer and the tumor microenvironment. Small GTPases 2015, 6:123-133

19. Zandvakili I, Lin Y, Morris JC, Zheng Y: Rho GTPases: anti- or proneoplastic targets? Oncogene 2017, 36:3213-3222

20. Smithers C, Overduin M: Structural mechanisms and drug discovery prospects of Rho GTPases. Cells 2016, 5:E26

21. Riching KM, Keely PJ: Rho family GTPases: making it to the third dimension. Int J Biochem Cell Biol 2015, 59:111-115

22. Li H, Peyrollier K, Kilic G, Brakebusch C: Rho GTPases and cancer. Biofactors 2014, 40:226-235

23. Zuo Y, Oh W, Ulu A, Frost JA: Minireview: mouse models of Rho GTPase function in mammary gland development, tumorigenesis, and metastasis. Mol Endocrinol 2016, 30:278-289

24. Schnelzer A, Prechtel D, Knaus U, Dehne K, Gerhard M, Graeff H, Harbeck N, Schmitt M, Lengyel E: Rac1 in human breast cancer: overexpression, mutation analysis, and characterization of a new isoform, Rac1b. Oncogene 2000, 19:3013-3020 
25. Fritz G, Brachetti C, Bahlmann F, Schmidt M, Kaina B: Rho GTPases in human breast tumours: expression and mutation analyses and correlation with clinical parameters. Br J Cancer 2002, 87:635-644

26. Tang Y, Olufemi L, Wang M-T, Nie D: Role of Rho GTPases in breast cancer. Front Biosci 2008, 13:759-776

27. Wertheimer E, Gutierrez-Uzquiza A, Rosemblit C, Lopez-Haber C, Sosa MS, Kazanietz MG: Rac signaling in breast cancer: a tale of GEFs and GAPs. Cell Signal 2012, 24:353-362

28. Yoshida T, Zhang Y, Rivera Rosado LA, Chen J, Khan T, Moon SY, Zhang B: Blockade of Rac1 activity induces G1 cell cycle arrest or apoptosis in breast cancer cells through downregulation of cyclin D1, survivin, and X-linked inhibitor of apoptosis protein. Mol Cancer Ther 2010, 9:1657-1668

29. Yan Y, Greer PM, Cao PT, Kolb RH, Cowan KH: RAC1 GTPase plays an important role in gamma-irradiation induced G2/M checkpoint activation. Breast Cancer Res 2012, 14:R60

30. Hein AL, Post CM, Sheinin YM, Lakshmanan I, Natarajan A, Enke CA, Batra SK, Ouellette MM, Yan Y: RAC1 GTPase promotes the survival of breast cancer cells in response to hyper-fractionated radiation treatment. Oncogene 2016, 35:6319-6329

31. Kikuchi K, Li X, Zheng Y, Takano Y: Invasion of breast cancer cells into collagen matrix requires TGF-alpha and Cdc42 signaling. FEBS Lett 2011, 585:286-290

32. Cardama GA, Comin MJ, Hornos L, Gonzalez N, Defelipe L, Turjanski AG, Alonso DF, Gomez DE, Menna PL: Preclinical development of novel Rac1-GEF signaling inhibitors using a rational design approach in highly aggressive breast cancer cell lines. Anticancer Agents Med Chem 2014, 14:840-851

33. Dokmanovic M, Hirsch DS, Shen Y, Wu WJ: Rac1 contributes to trastuzumab resistance of breast cancer cells: rac1 as a potential therapeutic target for the treatment of trastuzumab-resistant breast cancer. Mol Cancer Ther 2009, 8:1557-1569

34. Gonzalez N, Cardama GA, Comin MJ, Segatori VI, Pifano M, Alonso DF, Gomez DE, Menna PL: Pharmacological inhibition of Rac1-PAK1 axis restores tamoxifen sensitivity in human resistant breast cancer cells. Cell Signal 2017, 30:154-161

35. Chen HY, Yang YM, Stevens BM, Noble M: Inhibition of redox/Fyn/c-Cbl pathway function by $\mathrm{Cdc} 42$ controls tumour initiation capacity and tamoxifen sensitivity in basal-like breast cancer cells. EMBO Mol Med 2013, 5:723-736

36. Montalvo-Ortiz BL, Castillo-Pichardo L, Hernández E, HumphriesBickley T, De la Mota-Peynado A, Cubano LA, Vlaar CP, Dharmawardhane S: Characterization of EHop-016, novel small molecule inhibitor of Rac GTPase. J Biol Chem 2012, 287:13228-13238

37. Lin Y, Zheng Y: Approaches of targeting Rho GTPases in cancer drug discovery. Expert Opin Drug Discov 2015, 10:991-1010

38. Lin EY, Jones JG, Li P, Zhu L, Whitney KD, Muller WJ, Pollard JW: Progression to malignancy in the polyoma middle $\mathrm{T}$ oncoprotein mouse breast cancer model provides a reliable model for human diseases. Am J Pathol 2003, 163:2113-2126

39. Walker MK, Boberg JR, Walsh MT, Wolf V, Trujillo A, Duke MS, Palme R, Felton LA: A less stressful alternative to oral gavage for pharmacological and toxicological studies in mice. Toxicol Appl Pharmacol 2012, 260:65-69

40. Committee for the Update of the Guide for the Care and Use of Laboratory Animals; National Research Council: Guide for the Care and Use of Laboratory Animals: Eighth Edition. Washington, DC, National Academies Press, 2011

41. Marjon NA, Hu C, Hathaway HJ, Prossnitz ER: G protein-coupled estrogen receptor regulates mammary tumorigenesis and metastasis. Mol Cancer Res 2014, 12:1644-1654

42. Zakharchenko O, Greenwood C, Alldridge L, Souchelnytskyi S: Optimized protocol for protein extraction from the breast tissue that is compatible with two-dimensional gel electrophoresis. Breast Cancer (Auckl) 2011, 5:37-42
43. Aldridge GM, Podrebarac DM, Greenough WT, Weiler IJ: The use of total protein stains as loading controls: an alternative to highabundance single-protein controls in semi-quantitative immunoblotting. J Neurosci Methods 2008, 172:250-254

44. Kazanietz MG, Caloca MJ: The Rac GTPase in cancer: from old concepts to new paradigms. Cancer Res 2017, 77:5445-5451

45. Lam BD, Hordijk PL: The Rac1 hypervariable region in targeting and signaling: a tail of many stories. Small GTPases 2013, 4:78-89

46. Hein SM, Haricharan S, Johnston AN, Toneff MJ, Reddy JP, Dong J, $\mathrm{Bu} \mathrm{W}, \mathrm{Li}$ Y: Luminal epithelial cells within the mammary gland can produce basal cells upon oncogenic stress. Oncogene 2016, 35:1461-1467

47. Kabos P, Haughian JM, Wang X, Dye WW, Finlayson C, Elias A, Horwitz KB, Sartorius CA: Cytokeratin 5 positive cells represent a steroid receptor negative and therapy resistant subpopulation in luminal breast cancers. Breast Cancer Res Treat 2011, 128:45-55

48. Alshareeda AT, Soria D, Garibaldi JM, Rakha E, Nolan C, Ellis IO, Green AR: Characteristics of basal cytokeratin expression in breast cancer. Breast Cancer Res Treat 2013, 139:23-37

49. Castillo-Pichardo L, Humphries-Bickley T, De La Parra C, ForestierRoman I, Martinez-Ferrer M, Hernandez E, Vlaar C, Ferrer-Acosta Y, Washington AV, Cubano LA, Rodriguez-Orengo J, Dharmawardhane S: The Rac inhibitor EHop-016 inhibits mammary tumor growth and metastasis in a nude mouse model. Transl Oncol 2014, 7:546-555

50. Strumane K, Rygiel T, van der Valk M, Collard JG: Tiam1-deficiency impairs mammary tumor formation in MMTV-c-neu but not in MMTV-c-myc mice. J Cancer Res Clin Oncol 2009, 135:69-80

51. Laurin M, Huber J, Pelletier A, Houalla T, Park M, Fukui Y, HaibeKains B, Muller WJ, Côté JF: Rac-specific guanine nucleotide exchange factor DOCK1 is a critical regulator of HER2-mediated breast cancer metastasis. Proc Natl Acad Sci U S A 2013, 110:7434-7439

52. Cheung KJ, Gabrielson E, Werb Z, Ewald AJ: Collective invasion in breast cancer requires a conserved basal epithelial program. Cell 2013, 155:1639-1651

53. Qin L, Liao L, Redmond A, Young L, Yuan Y, Chen H, O'Malley BW, $\mathrm{Xu} \mathrm{J}$ : The AIB1 oncogene promotes breast cancer metastasis by activation of PEA3-mediated matrix metalloproteinase 2 (MMP2) and MMP9 expression. Mol Cell Biol 2008, 28:5937-5950

54. Flowers M, Schroeder JA, Borowsky AD, Besselsen DG, Thomson CA, Pandey R, Thompson PA: Pilot study on the effects of dietary conjugated linoleic acid on tumorigenesis and gene expression in PyMT transgenic mice. Carcinogenesis 2010, 31:1642-1649

55. Johansson J, Berg T, Kurzejamska E, Pang MF, Tabor V, Jansson M, Roswall P, Pietras K, Sund M, Religa P, Fuxe J: MiR-155-mediated loss of C/EBPbeta shifts the TGF-beta response from growth inhibition to epithelial-mesenchymal transition, invasion and metastasis in breast cancer. Oncogene 2013, 32:5614-5624

56. Shahi P, Slorach EM, Wang CY, Chou J, Lu A, Ruderisch A, Werb Z: The transcriptional repressor ZNF503/Zeppo2 promotes mammary epithelial cell proliferation and enhances cell invasion. J Biol Chem 2015, 290:3803-3813

57. Martin TA, Ye L, Sanders AJ, Lane J, Jiang WG: Cancer invasion and metastasis: molecular and cellular perspective. Edited by Jandial R. In Metastatic Cancer: Clinical and Biological Perspectives. Austin, TX: Landes Bioscience, 2013. pp. 135-168

58. Arias-Romero LE, Chernoff J: Targeting Cdc42 in cancer. Expert Opin Ther Targets 2013, 17:1263-1273

59. Guo Y, Kenney SR, Cook LS, Adams SF, Rutledge T, Romero E, Oprea T, Sklar LA, Bedrick E, Wiggins CL, Kang H, Lomo L, Muller CY, Wandinger-Ness A, Hudson LG: A novel pharmacologic activity of ketorolac for therapeutic benefit in ovarian cancer patients. Clin Cancer Res 2015, 21:5064-5072

60. Warner TD, Giuliano F, Vojnovic I, Bukasa A, Mitchell JA, Vane JR: Nonsteroid drug selectivities for cyclo-oxygenase-1 rather than cyclooxygenase- 2 are associated with human gastrointestinal toxicity: a full in vitro analysis. Proc Natl Acad Sci U S A 1999, 96:7563-7568 